\title{
Activation of Factor XII-dependent Pathways in Human Plasma by Hematin and Protoporphyrin
}

\author{
Cart G. Becker, Mary Wagner, Alan P. Kaplan, Michael Silverberg, Robert W. Grady, \\ Heng Liem, and Ursula Muller-Eberhard \\ Departments of Pathology and Pediatrics, The Cornell Thrombosis Specialized Center of Research, The New York Hospital-Cornell \\ Medical Center, New York City 10021; Division of Allergy, Rheumatology and Clinical Immunology, State University \\ of New York Health Sciences Center, Stony Brook, New York 11794
}

\begin{abstract}
Intravenous administration of hematin is effective in the treatment of acute exacerbations of the inducible porphyrias. In the course of such treatment, coagulopathies have occurred that are characterized by prolongation of prothrombin time, partial thromboplastin time, and formation of fibrin split products. In experiments in vitro with normal human plasma, we observed that hematin and protoporphyrin activated Factor XII-dependent pathways of coagulation and fibrinolysis, and that they generated kallikrein activity. Incubation of protoporphyrin with purified Factor XII resulted in activation as measured by amidolysis of a chromogenic substrate. Neither coproporphyrin, uroporphyrin, $\delta$-aminolevulinic acid, porphobilinogen, or bilirubin activated Factor XII-dependent pathways. Exposure of serum containing added uroporphyrin, coproporphyrin, and protoporphyrin, but not hematin, to ultraviolet light $(405 \mathrm{~nm})$ resulted in activation of the classical pathway of the complement system. On the other hand, exposure of plasma containing uroporphyrin or coproporphyrin to ultraviolet light did not result in activation of Factor XII-dependent pathways.
\end{abstract}

\section{Introduction}

The porphyrias are a group of disorders due to deficiencies of various enzymes in the biosynthetic pathway of heme. They are characterized by accumulation of heme precursors, which lead to a wide variety of sequelae, especially in the brain, liver, and skin, which occur via largely undefined mechanisms (1, 2). Injury to skin, particularly after solar exposure, is characteristic of congenital erythropoietic porphyria, protoporphyria, and porphyria cutanea tarda. Recent data suggest that the skin changes may be due to activation of the classical pathway of the complement system (3-6). Another group of porphyrias, the inducible porphyrias, is usually not associated with skin lesions. These patients profit from hematin injections in periods of exacerbations of their disease (7). In some patients, administration of hematin has been associated with prolongation of prothrombin time, partial thromboplastin time (PTT), and evidence of fibrinolysis (8). These observations suggested to us that hematin and perhaps other porphyrin compounds might

Address reprint requests to Dr. Becker, Department of Pathology, Cornell University Medical College. 1985.

Received for publication 30 July 1984 and in revised form 1 April

J. Clin. Invest.

(C) The American Society for Clinical Investigation, Inc.

0021-9738/85/08/0413/07 \$1.00

Volume 76, August 1985, 413-419 activate Factor XII-dependent pathways of coagulation and fibrinolysis and might generate kinin. In the in vitro experiments described below, we observed that hematin and protoporphyrin activated the contact system in normal human plasma, while uroporphyrin, coproporphyrin, $\delta$-aminolevulinic acid, porphobilinogen, and bilirubin did not. These data indicate that activation of Factor XII-dependent pathways is a potential risk associated with hematin administration, and that activation of these pathways may contribute to tissue injury in those cutaneous porphyrias in which protoporphyrin accumulates.

\section{Methods}

Porphyrins. Hematin was obtained in crystalline form from Sigma Chemical Co., St. Louis, MO. Protoprophyrin, coproporphyrin, uroporphyrin, $\delta$-aminolevulinic acid, porphobilinogen, and bilirubin were obtained from Porphyrin Products, Logan, UT. According to the manufacturer, uroporphyrin and coproporphyrin were $>97 \%$ pure as measured by spectral analysis and high performance liquid chromatography (HPLC); ${ }^{1}$ crystalline protoporphyrin was homogeneous when analyzed by thin-layer chromatography (9). The porphyrins and related compounds were dissolved in $0.1 \mathrm{~N} \mathrm{NaOH}$ and then diluted to working concentrations by addition of phosphate-buffered physiologic saline (PBS, $0.15 \mathrm{M} \mathrm{NaCl}, 0.01 \mathrm{M}$ sodium-potassium phosphate; $\mathrm{pH}$ 7.4), the final pH being readjusted to 7.4 with $0.1 \mathrm{~N} \mathrm{HCl}$. The actual concentration of these substances was determined spectrophotometrically (10). All solutions were prepared fresh each day and kept on ice in foil-wrapped plastic tubes for not $>2 \mathrm{~h}$ before use.

Reagents for measurement of Factor XII-dependent pathways. Celite (Filter Aid) was obtained from Fisher Scientific Co., Springfield, NJ. Rabbit brain cephalin, bovine thrombin, and Escherichia coli endotoxin (lipopolysaccharide [LPS]) from serotype 0111.B4 were obtained from Sigma Chemical Co. Bz-pro-phe-arg-p-nitroanilide was obtained from Vega Biochemicals, Tucson, AZ. Human plasma that was genetically deficient in Factor XII, high molecular weight kininogen (HMK), and prekallikrein (PK) were obtained from George King Biomedical Inc., Overland Park, KS. Limulus amoebocyte lysate assay kits were obtained from M.A. Bioproducts, Walkersville, MD. Polymyxin B (PB) was obtained through the courtesy of Burroughs Wellcome Co., Research Triangle Park, NC. Ellagic acid was obtained from Aldrich Chemical Co., Milwaukee, WI. It was brought into solution in the same manner as the porphyrins. No visible precipitate was found on standing after neutralization.

Complement reagents. Rabbit antiserum to human $\mathrm{C}_{3}$ was obtained from American Hoechst Corp., Somerville, NJ.

Plasma. Normal human plasma was obtained from laboratory

1. Abbreviations used in this paper: CHFI, corn Hageman factor inhibitor; ECLT, euglobulin clot lysis time; HF, Hageman factor; HMK, high molecular weight kininogen; HPLC, high performance liquid chromatography; LBTI, lima bean trypsin inhibitor, LPS, lipopolysaccharide; $\mathrm{PB}$, polymyxin B; PBS, phosphate-buffered physiologic saline; PK, prekallikrein; PTT, partial thromboplastin time; PPACMK, D-pro-phe-arg-chloromethylketone; SBTI, soybean trypsin inhibitor. 
personnel. Blood was anticoagulated with sodium citrate in polypropylene tubes. The blood was centrifuged at $9,000 \mathrm{~g}$ for $10 \mathrm{~min}$ and the plasma pipetted with polypropylene pipette tips (Eppendorff; Brinkmann Instruments, Westbury, NY) to other polypropylene tubes, centrifuged again at $9,000 \mathrm{~g}$ for $10 \mathrm{~min}$, and then pipetted into polypropylene tubes (Nunc, Roskilde, Denmark) and stored at $-70^{\circ} \mathrm{C}$. Serum from the same donors was obtained from blood clotted in glass tubes and stored in polypropylene tubes at $-70^{\circ} \mathrm{C}$.

\section{Measurement of the effect of hematin and its precursors on activation of Factor XII-dependent pathways}

$P T T$. The effect of hematin, protoporphyrin, coproporphyrin, uroporphyrin, $\delta$-aminolevulinic acid, porphobilinogen, and bilirubin on the partial thromboplastin time of normal human citrated plasma was compared with that of suspensions of celite in PBS or PBS alone. The studies were done in polyethylene cups (Fisher Scientific Co.) using a fibrometer (Baltimore Biological Laboratories, Baltimore, MD) as described previously (11). In brief, $100 \mu \mathrm{l}$ of plasma were warmed to $37^{\circ} \mathrm{C}$ for $60 \mathrm{~s}$ after which $100 \mu \mathrm{l}$ of test or control substance was added and the mixture incubated at $37^{\circ} \mathrm{C}$ for $60 \mathrm{~s} .50 \mu \mathrm{l}$ of $0.05 \mathrm{M}$ $\mathrm{CaCl}_{2}$ in $0.05 \mathrm{M}$ glyoxalline- $0.1 \mathrm{M} \mathrm{NaCl}$ buffer (Sigma Chemical Co.), pH 7.3, and $50 \mu \mathrm{l}$ of cephalin suspended in PBS were added simultaneously and the clot timer started. Polypropylene pipette tips were used in all assays. Assays were performed in triplicate; the coefficient of variation was $<5 \%$. Data shown represent the mean of three determinations.

Euglobulin clot lysis time (ECLT). The effect of the above substances on ECLT was measured according to a modification of the technique of Ogston et al. (12). $100 \mu \mathrm{l}$ of a dilution of test substance was added to $200 \mu \mathrm{l}$ of plasma in $10 \times 75-\mathrm{mm}$ polystyrene test tubes (Pharmacia Fine Chemicals, Piscataway, NJ). To these were added $4 \mathrm{ml}$ of cold

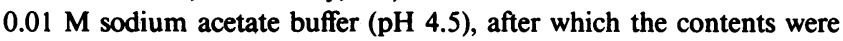
mixed and incubated at $37^{\circ} \mathrm{C}$ for $30 \mathrm{~min}$. The tubes were centrifuged for $5 \mathrm{~min}$ in a serofuge (Clay Adams, Inc., New York City), and the supernates were thoroughly decanted by inversion of the tubes and blotting on paper towels. The euglobulin precipitates were redissolved

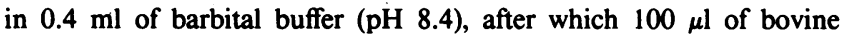
fibrinogen $(4 \mathrm{mg} / \mathrm{ml})$ and $50 \mu \mathrm{l}$ of bovine thrombin $(50 \mathrm{U} / \mathrm{ml})$ were added. The tubes were then incubated in a water bath at $37^{\circ} \mathrm{C}$ and inverted every $5 \mathrm{~min}$ to monitor clot lysis. The lysis time was taken as the time at which the clot was sufficiently lysed to run out of the tube on inversion. All determinations were performed in duplicate; the clot lysis times of duplicate samples were within $5 \mathrm{~min}$ of each other. Data shown represent the mean of two values.

Activation of purified Factor XII by protoporphyrin. Factor XII was purified from human plasma as described previously (13). The specific activity of the Hageman factor (HF [Factor XII]) preparation was 30 $\mathrm{U} / \mathrm{mg}$. It gave a single band of molecular weight $(80,000)$ on SDS polyacrylamide gel electrophoresis (nonreduced); on reduction, $\sim 5 \%$ was cleaved. One preparation of Hageman factor was used.

Chromogenic substrates S2302 (H-D-pro-phe-arg-p-nitroanilide) and S2222 ( $N$-benzoyl-ile-glu-gly-arg- $p$-nitroanilide) were obtained from Helena Laboratories, Beaumont, TX. D-phe-pro-arg-chloromethylketone (PPACMK) was obtained from Calbiochem-Behring Corp., La Jolla, CA.

Amidolytic assays of Factor XII activity were conducted at $37^{\circ} \mathrm{C}$ in a volume of $400 \mu \mathrm{l}$. The assay reagent was $0.05 \mathrm{M}$ Tris/ $\mathrm{HCl}, \mathrm{pH} 7.8$, containing $0.002 \%$ Triton $\mathrm{X}-100$ and $0.5 \mathrm{mM}$ chromogenic substrate, either S2302 or S2222. The absorbance at $405 \mathrm{~nm}$ was recorded with time on a Gilford model 250 spectrophotometer. To measure the effect of protoporphyrin on autoactivation of Factor XII HF, preparations were first treated with $5 \mu \mathrm{M}$ PPACMK for $60 \mathrm{~min}$ at $37^{\circ} \mathrm{C}$. The chloromethyl ketone ester was used to destroy traces of activated HF in the preparation; the subsequent dilution in the cuvette then allowed autoactivation to be recorded from a baseline. For the time course of activation, the protein was diluted $1: 10$ into $50 \mathrm{mM}$ Tris/HCl, $\mathrm{pH} 7.5$, containing $0.001 \%$ Triton $\mathrm{X}-100$ and $0.038 \mathrm{mM}$ protoporphyrin. The control had no protoporphyrin added. The evolution of enzymatic

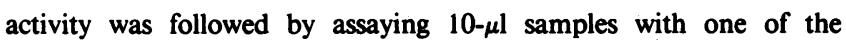
chromogenic substrates described above. The effect of hematin, coproporphyrin, and uroporphyrin on purified Factor XII could not be measured because their colors interfered with spectrophotometric measurement of amidolysis.

Kallikrein-like activity. In this assay, $0.1 \mathrm{ml}$ of normal human plasma was incubated for $10 \mathrm{~min}$ at $37^{\circ} \mathrm{C}$ with a test substance or buffer control in $0.1 \mathrm{ml}$ of $0.05 \mathrm{M}$ Tris/ $\mathrm{HCl}$ buffer ( $\mathrm{pH} 7.8$ ) brought to ionic strength $(0.05 \mu)$ with $\mathrm{NaCl}$, and an additional $0.1 \mathrm{ml}$ of Tris/ $\mathrm{HCl}$ buffer. An aliquot $(0.03 \mathrm{ml})$ of the same buffer was then added, and the reaction mixture transferred to a siliconized quartz cuvette coated with Prosil-28 (PCR, Inc., Gainesville, FL) containing $0.1 \mathrm{ml}$ of Bz-pro-phe-arg-p-nitroanilide. In other experiments, $0.1 \mathrm{ml}$ of plasma in $0.8 \mathrm{ml}$ of buffer was incubated with $0.1 \mathrm{ml}$ of a solution of test substance and then transferred to a cuvette containing $0.1 \mathrm{ml}$ of substrate in $1.4 \mathrm{ml}$ of $0.05 \mathrm{M}$ Tris/HCl. The absorbance at $405 \mathrm{~nm}$ was recorded every minute for $10 \mathrm{~min}$ in a Zeiss spectrophotometer, while the temperature of the cuvette was being maintained at $37^{\circ} \mathrm{C}$. All assays were performed in duplicate. Various dilutions of $p$-nitroaniline (Sigma Chemical Co.) dissolved in Tris/ $\mathrm{HCl}$ buffer served as reference; the standard curve was linear from 0.5 to $50 \mathrm{nmol}$ (14). The extinction coefficient for $p$-nitroaniline in Tris buffer at $405 \mathrm{~nm}$ (Zeiss spectrophotometer M4QII), Slit $0.02 \mathrm{~mm}$; was $8,443 \mathrm{~mol} /$ liter per $\mathrm{cm}$. To demonstrate the specificity of Bz-pro-phe-arg-p-nitroanilide for kallikrein, duplicate assays were performed with either lima bean trypsin inhibitor (LBTI; $100 \mu \mathrm{g}$ ) or soybean trypsin inhibitor (SBTI; $20 \mu \mathrm{g}$ ) being added to the incubation mixture $1 \mathrm{~min}$ before addition of the substrate. The effect of the Factor XII inhibitor purified from popcorn according to the technique of Hojima et al. (15) was also measured. The HF inhibitor from corn (CHFI) with a pI of 6.1 was used. Plasma from patients genetically deficient in Factor XII, prekallikrein, or HMK was also challenged with the test substances, and its amidolytic activity was measured. Partial thromboplastin times, ECLTs, and measurements of kallikrein activity were performed simultaneously on aliquots of freshly thawed plasma employing freshly made solution of the test substances. Experiments in which kallikrein activity was measured, with the exception of the dose-response curves, were performed three times and the data pooled for statistical comparisons. The latter were performed using the two-tailed $t$ test (16).

Measurement of the effect of porphyrins on the complement activity of normal plasma and serum. $100 \mu \mathrm{l}$ of solutions of test substances or buffer (control) were mixed in sterile plastic petri dishes with $500 \mu \mathrm{l}$ of serum or citrated plasma reconstituted by addition of $\mathrm{CaCl}_{2}$ and $\mathrm{MgCl}_{2}$ and placed on ice. Dishes were kept covered with aluminum foil or exposed to ultraviolet light $(405 \mathrm{~nm})$ at a distance of $5 \mathrm{~cm}$ for $60 \mathrm{~min}$. Samples were taken directly from these mixtures and total hemolytic complement activity was measured according to the radial hemolysis assay of Truedsson et al. (17), either immediately or $30 \mathrm{~min}$ after incubation at $37^{\circ} \mathrm{C}$. Plates were layered with agarose $(0.8 \%)$ containing $\mathrm{Ca}^{2+}, \mathrm{Mg}^{2+}$, and sheep erythrocytes sensitized with rabbit antibody, after which wells $6 \mathrm{~mm}$ in diameter were cut in the agar. A standard curve was prepared by pipetting $50 \mu \mathrm{l}$ of serial, twofold dilutions of normal human plasma reconstituted with respect to $\mathrm{Ca}^{2+}$ and $\mathrm{Mg}^{2+}$ into duplicate wells. The plates were refrigerated at $4^{\circ} \mathrm{C}$ overnight and then incubated at $37^{\circ} \mathrm{C}$ for $2 \mathrm{~h}$. Measurements of the zones of hemolysis were made with calipers. When the area of hemolysis was plotted against the log of the reciprocal of the dilution of serum or plasma and the data analyzed by the method of least squares, a linear relationship was observed, ( $r$ values of these slopes ranged between 0.96 and $0.98 ; t$ tests of these values demonstrated significance, $P<0.01$ ). The coefficient of variation of duplicate samples was $3 \%$. Y intercepts were calculated and the consumption of hemolytic activity relative to that of the controls was determined.

Heating serum or plasma samples to $56^{\circ} \mathrm{C}$ for $30 \mathrm{~min}$ abolished hemolytic activity. Chelation of plasma or serum with EDTA or EGTA before incubation with uroporphyrin or coproporphyrin and subsequent irradiation abolished the consumption of complement. In these assays, no differences were observed between the responses of 
serum and plasma to substances capable of activating the classical complement pathway.

In subsequent experiments, citrated plasma samples were reconstituted with $\mathrm{Ca}^{2+}$ and $\mathrm{Mg}^{2+}$ and these incubated with the test substances or buffer (control) for $60 \mathrm{~min}$ as previously described, with and without irradiation. Samples of these mixtures were taken and assayed for complement activity, partial thromboplastin time, kallikrein activity, and fibrinolytic activity, as described above. In addition, samples were subjected to crossed immunoelectrophoresis using rabbit antisera to human $\mathrm{C}_{3}$ to determine if cleavage of $\mathrm{C}_{3}$ had occurred (18).

Assay of endotoxin activity. Hematin and protoporphyrin solutions were tested for bacterial endotoxin activity using the Limulus amoebocyte lysate assay. Both substances were found to interfere with the assay. To eliminate endotoxin contamination as a cause of the observed activation of Factor XII-dependent pathways by these substances, two experiments were performed. In the first experiment, hematin and protoporphyrin were incubated with varying concentrations of $\mathrm{PB}$, a cationic detergent that binds to and inhibits the capacity of bacterial LPS to release interleukin-1 from monocytes (19). The effect of PB on the ability of hematin and protoporphyrin to activate Factor XIIdependent pathways was measured using the assay of kallikrein activity described above. In the second experiment, samples of hematin, protoporphyrin, and endotoxin derived from $E$. coli were heated to $180^{\circ} \mathrm{C}$ for $4 \mathrm{~h}$ and then compared with unheated samples with respect to their ability to generate kallikrein activity.

\section{Results}

Effect of hematin and various porphyrins on Factor XIIdependent pathways. It was observed that hematin and protoporphyrin caused a decrease in the unactivated PTT and the ECLT (Table I), while $\delta$-aminolevulinic acid, bilirubin, uroporphyrin, and coproporphyrin did not induce activation of these Factor XII-dependent pathways.

It was also observed that protoporphyrin and hematin were capable of generating kallikrein activity in normal human plasma, while coproporphyrin and uroporphyrin did not. Generation of kallikrein activity by protoporphyrin and hematin was dose dependent (Fig. 1), describing logarithmic curves with the formula $y=3.9937+7.8338 \ln x$, and $y=-0.3013$ $+3.214 \ln x$, respectively. The $r$ values for these curves were 0.9969 and 0.9769 , respectively, and were highly significant $(P$ $<0.005$ ). Neither $\delta$-aminolevulinic acid nor bilirubin generated kallikrein activity (not shown). PBS added to plasma generated $1 \pm 0.1997 \mathrm{nmol}$ of $p$-nitroaniline per milliliter per minute.

To demonstrate conclusively that the observations described were due to activation of Factor XII-dependent pathways, two additional experiments were performed. In the first experiment,

Table I. Activation of Factor XII-dependent Pathways

\begin{tabular}{lllr}
\hline Substance & $\begin{array}{l}\text { Quantity added to } \\
\text { reaction mixture }\end{array}$ & $\begin{array}{l}\text { Unactivated } \\
\text { PTT }\end{array}$ & ECLT \\
\hline & $n m o l$ & $s$ & $\min$ \\
Hematin & 3.0 & 323 & 40 \\
Protoporphyrin & 4.9 & 167 & 30 \\
Uroporphyrin & 6.6 & 450 & $\geq 180$ \\
Coproporphyrin & 4.9 & 474 & $\geq 180$ \\
Bilirubin & 4.3 & 500 & $\geq 180$ \\
D-Amino-levulinic acid & 3.6 & 480 & $\geq 180$ \\
PBS & - & 461 & $\geq 180$ \\
Celite & $2 \mathrm{mg} / \mathrm{ml}$ & 97 & 20 \\
\hline
\end{tabular}

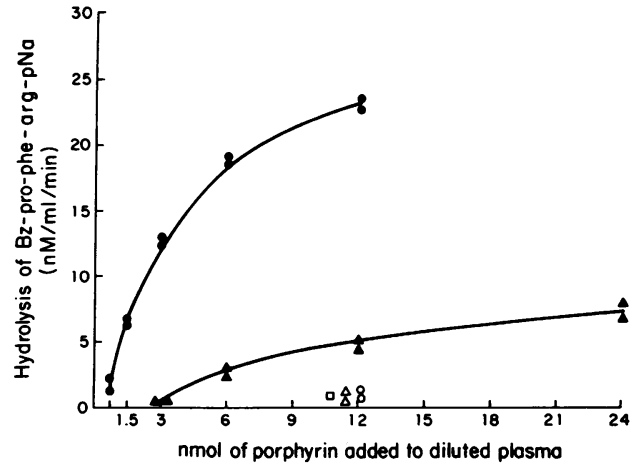

Figure 1. The effect of varying concentrations of protoporphyrin and hematin on the generation of kallikrein activity in normal human plasma. ๑, protoporphyrin IX; $\Delta$, hematin; O, coproporphyrin III; $\Delta$, uroporphyrin I; 口, PBS.

it was determined that the generation of kallikrein activity that occurred when normal plasma was mixed with hematin and protoporphyrin did not occur when plasma was deficient in Factor XII, prekallikrein, or HMK (Table II). These differences were statistically significant $(P<0.001)$. In the second experiment, it was observed that CHFI, which is known to inhibit Factor XII and not kallikrein (15), and SBTI, but not LBTI, inhibited the generation of kallikrein activity by hematin and protoporphyrin (Table III). These differences were also statistically significant $(P<0.001)$.

As can be seen in Fig. 2, incubation of purified HF with protoporphyrin leads to activation as assessed by using a synthetic chromogenic substrate. Since effective surfaces accomplish this by catalyzing an activation reaction $(12,20-23)$, we next assessed whether HF would autoactivate in plastic test tubes when exposed to protoporphyrin. Fig. 3 is an autoactivation curve which demonstrates that exposure to glass or protoporphyrin leads to activation (curves $B$ and $C$ ), while plastic does not (curve A). Addition of protoporphyrin to the chromogenic substrates in the absence of plasma did not induce amidolysis. Autoactivation of HF is not seen in plastic tubes in the absence of polybrene (13).

Bacterial LPS is known to activate Factor XII-dependent pathways (24). Therefore, experiments were performed (Table IV) to eliminate contamination of hematin and protoporphyrin preparations by LPS as a cause of the observations described in Tables I, II, and III. In these experiments, hematin, protoporphyrin, ellagic acid, and LPS 0111/B4 were added to plasma before and after heating to $180^{\circ} \mathrm{C}$ for $4 \mathrm{~h}$, which was

Table II. Effect of Hematin and Protoporphyrin on Amidolysis* of Bz-pro-phe-arg-p-nitroanilide in Normal and Deficient Plasma

\begin{tabular}{llllll}
\hline & $\begin{array}{l}\text { Quantity added } \\
\text { to reaction } \\
\text { mixture }\end{array}$ & Normal & $\begin{array}{l}\text { XII } \\
\text { Deficient }\end{array}$ & $\begin{array}{l}\text { PK } \\
\text { Deficient }\end{array}$ & $\begin{array}{l}\text { HMK } \\
\text { Deficient }\end{array}$ \\
\hline & $n m o l$ & & & & \\
Hematin & 12 & 10.2 & 1.8 & 1.7 & 1.4 \\
Protoporphyrin & 3 & 14.2 & 1.2 & 1.5 & 2.5 \\
PBS & & 0.9 & 0.5 & 1.1 & 1.0 \\
\hline
\end{tabular}

* Nanomoles of $p$-nitroaniline generated per milliliter of plasma per minute. 
Table III. Effect of Protease Inhibitors on Activation of Amidolysis* of Bz-pro-phe-arg-p-nitroanilide by Hematin and Protoporphyrin

\begin{tabular}{lllllll}
\hline & $\begin{array}{l}\text { Quantity added } \\
\text { to reaction } \\
\text { mixture }\end{array}$ & $\begin{array}{l}\text { Control } \\
\text { Substance }\end{array}$ & PBS & CHFI & SBTI & LBTI \\
\hline & $n$ Inhibitor & \\
Hematin & 12 & & & & \\
Protoporphyrin & 3 & 10.2 & 2.0 & 1.4 & 12.5 \\
& & 14.2 & 2.3 & 1.9 & 15.4 \\
\hline
\end{tabular}

* Nanomoles of $p$-nitroaniline generated per milliliter of plasma per minute.

a treatment known to destroy LPS. It was seen that heating does not diminish the capacity of hematin, protoporphyrin, or ellagic acid to generate kallikrein activity, whereas it significantly inhibits the capacity of LPS to do so $(0.001<P<0.01)$.

Because PB has been shown to inhibit the capacity of LPS to generate interleukin-1 from monocytes (19), its effect on the capacity of hematin, protoporphyrin, and ellagic acid to generate kallikrein activity in human plasma was measured. PB is a cationic polypeptide that binds to LPS and, presumably, by inhibiting its negative charge, inhibits its activity. In all cases, the generation of kallikrein activity in human plasma was significantly inhibited (Table V; $P<0.001$ for each comparison). This effect, in view of the results of experiments described in Table IV, suggested that PB counteracts the negative charge on hematin, protoporphyrin, and ellagic acid, which appears to be essential for their activation of Factor XII-dependent pathways.

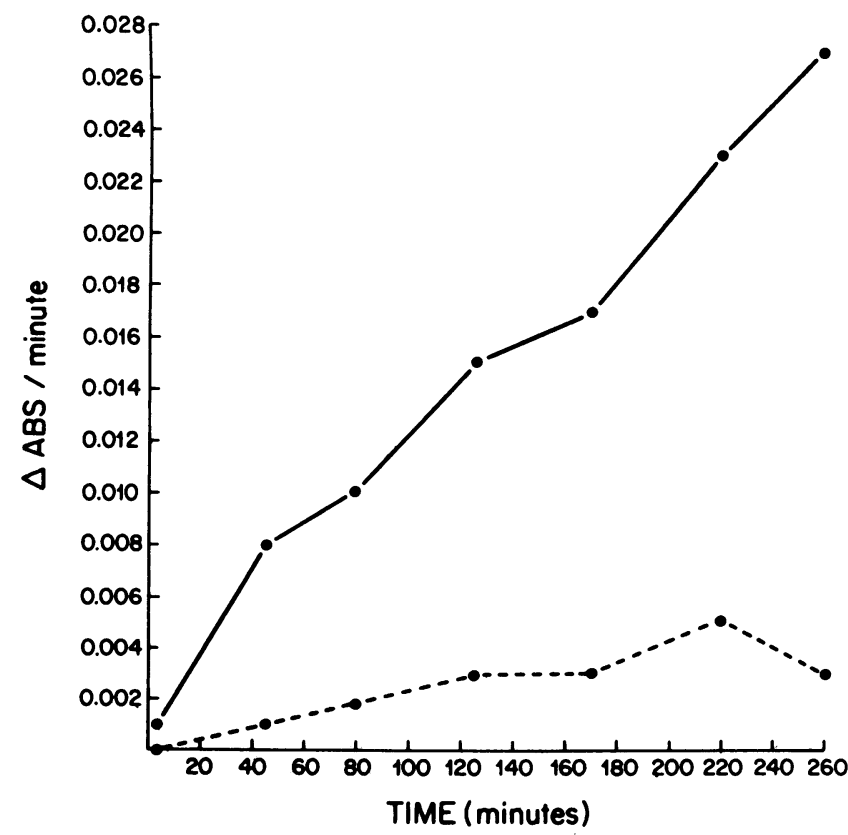

Figure 2. Activation of $\mathrm{HF}$ in the presence of protoporphyrin/ PPACMK-treated HF $(78.6 \mu \mathrm{g} / \mathrm{ml})$ incubated in plastic vials, with or without $0.038 \mathrm{mM}$ protoporphyrin at $37^{\circ} \mathrm{C}$. At intervals, $10-\mu \mathrm{l} \mathrm{sam}$ ples were withdrawn for assay with $\mathbf{S 2 3 0 2}$. The activity measured as the change in absorbance at $405 \mathrm{~nm} / \mathrm{min}(\triangle \mathrm{ABS} / \mathrm{min})$ plotted as a function of the time of incubation. proto IX $+\mathrm{HF}(\bullet-\bullet)$, proto-

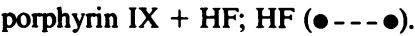

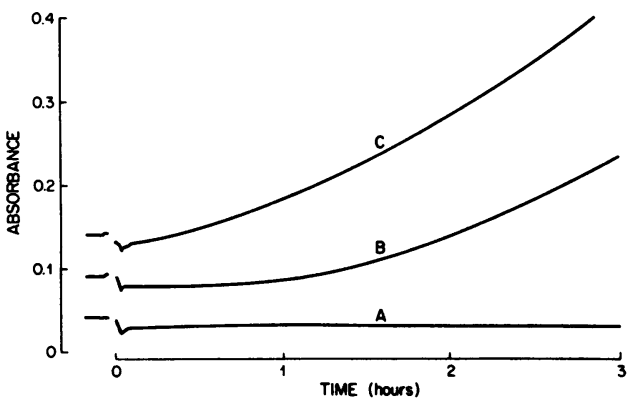

Figure 3. Autoactivation of HF: $9.7 \mu \mathrm{g} / \mathrm{ml}$ of PPACMK-treated HF were incubated in enzyme assay buffer containing $0.5 \mathrm{mM} \mathrm{S} 2222$. Curve A was recorded from a plastic cuvette containing $50 \mu \mathrm{g} / \mathrm{ml}$ polybrene. Curve B was recorded from a similar cuvette with the addition of $0.0019 \mathrm{mM}$ protoporphyrin. Curve $C$ was recorded from a glass cuvette without any additions.

Effect of irradiation with long wave ultraviolet light on activation of the classical complement pathways in serum containing prophyrin compounds. When either uroporphyrin, coproporphyrin, or protoporphyrin was mixed with serum and exposed to long wave ultraviolet light, the hemolytic complement activity decreased (Table VI). This decrease could be inhibited by EGTA or EDTA, which indicated that the classical complement pathways had been activated. Activation of this pathway was associated with cleavage of $\mathrm{C}_{3}$ as demonstrated by crossed immunoelectrophoresis. Protoporphyrin induced the activation of the classical pathway to a lesser extent than uroporphyrin or coproporphyrin, and required longer periods of exposure to ultraviolet light. In contrast, when hematin was mixed with serum and exposed to ultraviolet light, no activation of complement was demonstrable.

Effect of irradiation with long wave ultraviolet light on activation of the classical complement pathway and Factor XII-dependent pathways in plasma containing porphyrins. Because irradiation of serum containing uroporphyrin or coproporphyrin with long wave ultraviolet light resulted in activation of the classical complement pathway, we questioned whether these substances would also acquire the capacity to activate Factor XII-dependent pathways when mixed with plasma and irradiated. In the experiments shown in Table VII, citrated human plasma was reconstituted by addition of $\mathrm{Ca}^{2+}$. To 800 -

Table IV. Effect of Heating* on the Capacity of Various Substances to Activate Amidolysis $\ddagger$ of Bz-pro-phe-arg-p-nitroanilide

\begin{tabular}{llrr}
\hline Substance & $\begin{array}{l}\text { Quantity added } \\
\text { to reaction } \\
\text { mixture }\end{array}$ & $\begin{array}{l}\text { Before } \\
\text { heating }\end{array}$ & $\begin{array}{l}\text { After } \\
\text { heating* }\end{array}$ \\
\hline & nmol & & \\
Hematin & 11.0 & 5.0 & 6.9 \\
Protoporphyrin & 17.2 & 26.8 & 21.3 \\
Ellagic Acid & 66 & 12.0 & 13.2 \\
LPS 0111B4 & 20 & 3.2 & 0.9 \\
PBS & & 0.9 & \\
\hline
\end{tabular}

* $180^{\circ} \mathrm{C}$ for $4 \mathrm{~h}$.

₹ Nanomoles of $p$-nitroanaline generated per milliliter of plasma per minute. 
Table $V$. Effect of PB on Activation of Amidolysis* of Bz-pro-phearg-p-nitroanilide by Hematin, Protoporphyrin, and Ellagic Acid

\begin{tabular}{llrr}
\hline Substance & $\begin{array}{l}\text { Quantity added to } \\
\text { reaction mixture }\end{array}$ & $\begin{array}{l}\text { With } \\
\text { PB }\end{array}$ & $\begin{array}{l}\text { Without } \\
\text { PB }\end{array}$ \\
\hline & $n m o l$ & & \\
Hematin & 11.2 & 0.9 & 5.0 \\
Protoporphyrin & 8.5 & 1.4 & 11.7 \\
Ellagic acid & 66 & 13.8 & 33.1 \\
PBS & & 0.9 & 0.8 \\
\hline
\end{tabular}

*Nanomoles of $p$-nitroaniline generated per milliliter of plasma per minute.

$\mu l$ aliquots of plasma were added $200 \mu$ l of a solution containing the test substances or buffer alone. The mixtures were exposed to long wave ultraviolet light or kept in the dark for $60 \mathrm{~min}$. Duplicate $200-\mu 1$ aliquots were assayed for fibrinolytic activity by measuring ECLT. Duplicate samples were also taken to measure kallikrein activity and hemolytic complement activity. It was not possible to measure PTT in this set of experiments because fibrin strands formed during the 60 -min incubation period in the recalcified plasma treated with either celite or protoporphyrin. It can be seen that irradiation of plasma containing either uroporphyrin or coproporphyrin did not result in activation of fibrinolysis or generation of kallikrein activity, but did result in consumption of complement. In contrast, addition of hematin or protoporphyrin to plasma resulted in activation of fibrinolysis and generation of kallikrein activity, with both processes being unaffected by ultraviolet irradiation. In this experiment, the generation of kallikrein activity was not further enhanced by irradiating plasma containing hematin or protoporphyrin, as might have been expected if irradiation was essential to the effects of these molecules on autoactivation of Factor XII. Addition of protoporphyrin and irradiation with $405 \mathrm{~nm}$ light probably did not result in demonstrable consumption of complement, because the incubation time was only $1 \mathrm{~h}$ (see Table VI).

\section{Discussion}

In this study we show hematin and protoporphyrin as the causes of activation of the Factor XII-dependent pathways of coagulation and fibrinolysis, as well as for the generation of

Table VI. Effect of Irradiation of Porphyrin and Serum Mixtures with $405 \mathrm{~nm}$ Light on Activation and Consumption of Components of the Classical Complement Pathway

\begin{tabular}{|c|c|c|c|c|c|}
\hline \multirow[b]{2}{*}{$\begin{array}{l}\text { Duration of } \\
\text { irradiation }\end{array}$} & \multicolumn{5}{|l|}{ Substance* } \\
\hline & $\begin{array}{l}\text { Uro- } \\
\text { porphyrin }\end{array}$ & $\begin{array}{l}\text { Copro- } \\
\text { porphyrin }\end{array}$ & $\begin{array}{l}\text { Proto- } \\
\text { porphyrin }\end{array}$ & Hematin & PBS \\
\hline \multicolumn{6}{|l|}{$h$} \\
\hline 1 & $14 \ddagger$ & $8 \ddagger$ & $12 \ddagger$ & $1 \neq$ & $0 \ddagger$ \\
\hline 2 & 20 & 50 & 13 & 0 & 0 \\
\hline 3 & 100 & 100 & 24 & 1 & 0 \\
\hline 4 & 100 & 100 & 43 & 0 & 0 \\
\hline 5 & 100 & 100 & 47 & 0 & 0 \\
\hline
\end{tabular}

* Quantity added to reaction equals $17 \mathrm{nmol}$.

¥ Percentage of hemolytic activity consumed.
Table VII. Comparison of Complement

Consumption, Fibrinolysis, and Killikrein Generation Induced by Porphyrin Compounds

\begin{tabular}{llllll}
\hline & $\begin{array}{l}\text { Quantity added } \\
\text { to reaction } \\
\text { mixture }\end{array}$ & $\begin{array}{l}\text { 405 nm } \\
\text { light }\end{array}$ & $\begin{array}{l}\text { \% Decrease in } \\
\text { complement } \\
\text { activity }\end{array}$ & ECLT & $\begin{array}{l}\text { Kallikrein } \\
\text { activity* }\end{array}$ \\
\hline \multirow{4}{*}{ Uroporphrince } & nmol & & & min & \\
& 38 & + & 31 & 120 & 1.52 \\
Coproporphyrin & 38 & - & 0 & 120 & 2.00 \\
& 36 & + & 28 & 120 & 1.64 \\
Protoporphyrin & 35 & - & 9 & 125 & 1.44 \\
& 35 & + & 0 & 30 & 4.24 \\
Hematin & 37 & - & 0 & 30 & 4.66 \\
& 37 & + & 0 & 30 & 5.14 \\
PBS & & - & 0 & 30 & 5.73 \\
& & + & 0 & 125 & 2.03 \\
& & - & 0 & 120 & 2.12 \\
\hline
\end{tabular}

* Amidolysis of Bz-pro-phe-arg-p-nitroanilide by human plasma measured in nanomoles per milliliter per minute.

kallikrein activity. Activation of Factor XII-dependent pathways by hematin and protoporphyrin was not demonstrable in plasma from humans who were genetically deficient in Factor XII, HMK, or prekallikrein. Activation of the contact system of normal human plasma by hematin and protoporphyrin was prevented in the presence of a Factor XII inhibitor purified from popcorn, while the kallikrein activity generated was inhibitable with SBTI. Protoporphyrin appeared to catalyze the autoactivation of purified human Factor XII. Hematin did not activate the classical pathway of the complement system when mixed with serum or plasma, and subsequently irradiated with long wave ultraviolet light.

These observations appear to conflict with those of Green et al. (25), who observed that hematin inhibited the clotting of bovine fibrinogen by bovine thrombin, inhibited the amidolysis of a synthetic substrate by human thrombin, reduced Factor VIII:C activity, inhibited activation of VIII:C by thrombin and inhibited clot lysis. They attributed their findings to the binding of hematin to proteins, which resulted in inhibition of their biologic functions. We cannot explain the conflicting results; however, it is possible that they are due to differences in methodology. We dissolved hematin and the other porphyrin compounds rapidly and used them soon after preparation. Greene et al. (25) dissolved hematin in carbonate buffer over a period of $24 \mathrm{~h}$. During this time, oxidation of hematin may have occurred. Further, we added hematin directly to plasma, whereas in many of the assays performed by Greene et al. (25), hematin was mixed with purified or partially purified (enriched) components. Lastly, these authors observed that albumin interfered with the capacity of hematin to inhibit thrombin. This suggests that the binding of hematin to proteins, e.g., albumin and/or hemopexin, in whole plasma may dilute any inhibitory effects which it may have, thereby permitting activation of, in this instance, Factor XII-dependent pathways.

In contrast, neither uroporphyrin nor coproporphyrin activated Factor XII-dependent pathways in normal human plasma. Uroporphyrin, protoporphyrin, and coproporphyrin 
did, however, activate the classical pathway of the complement system when mixed with serum or plasma and exposed to long wave ultraviolet light. It was previously shown that activation of the classical pathway of the complement system occurred after addition of exogenous uroporphyrin or protoporphyrin to serum and subsequent exposure to long wave ultraviolet light, as well as after exposure of serum from patients with erythropoietic protoporphyria or porphyria cutanea tarda to long wave ultraviolet light (3-5). In the latter studies, the consumption of complement components was observed to correlate with the concentration of protoporphyrin in the serum. In more recent studies (26) it was shown that exposure of serum containing demethylchlortetracycline to long wave ultraviolet light also resulted in activation of the classical pathway. In our experiments, the capacity of protoporphyrin to activate the classical pathway of the complement system was less than that of uroporphyrin and coproporphyrin, and required longer periods of exposure to ultraviolet light. This finding suggests that the structural requirements for activation of Factor XII by porphyrins differ from those needed to activate the classical pathway of the complement system. The functional groups present on the porphyrin nucleus may differentially modulate its ability to activate one or more of the pathways studied. The charge associated with each molecule is significantly different (1). Uroporphyrin, for instance, contains eight carboxyl groups, while coproporphyrin has four, and both protoporphyrin and hematin have only two. Accordingly, the overall charge of these molecules can be significantly different, and hence their interaction with a specific protein or active site could differ considerably. Thus, one might expect the more negatively charged uroporphyrin molecule to interact more strongly with a hydrophilic environment than protoporphyrin. Furthermore, to the extent that contact between proteins is determined by electrostatic interactions, charged molecules that alter the net surface charge of a protein may enhance/ inhibit a given interaction. Cationic detergents, such as polymyxin B, which counteract negatively charged molecules, would be expected to reduce the effects seen.

In those situations where activation by ultraviolet light is required, the charge on the side chains also seems to play an important role. Since the carboxyl groups on none of the porphyrins studied are in conjugation with the tetrapyrrole nucleus, a direct interaction of the carboxyl groups and ultraviolet light seems unlikely. On the other hand, the course of subsequent reactions due to light energy absorbed by these molecules could be influenced by the degree to which the carboxyl groups cause electrostatic attraction/repulsion at the active sites concerned. For instance, photooxidation due to rose bengal, a highly conjugated molecule having only a single carboxyl group, has been shown to destroy the heme binding site of hemopexin (27). In our studies, those molecules possessing the most charge (uroporphyrin and coproporphyrin) appear to be the most active. Thus, tight binding to the active site may allow for maximal transfer of the energy stored in the porphyrin nucleus to the protein. That this situation is completely different from activation of Factor XII-dependent pathways is shown by the fact that ultraviolet light did not activate uroporphyrin and coproporphyrin vis-a-vis the latter pathways. The difference in the observations could also be explained by considering the aggregation state of the porphyrins concerned. Uroporphyrin remains in aqueous solution, protoporphyrin is highly aggregated, while coproporphyrin forms an intermediate state of aggregation. Depending upon the particular aggregation state of a porphyrin, it may interact differentially with the active site of a given protein, and thereby activate/inhibit under different conditions. In this connection, spectral analysis of the hematin and protoporphyrin at the concentrations used in assays described above indicated that they were aggregated, while coproporphyrin and uroporphyrin were not (28). The capacity of hematin and protoporphyrin to aggregate may cause them to form a negative surface of sufficient size to support activation of Factor XII.

Hematin represents a special case. While the nucleus of the other porphyrins studied is neutral, that of hematin is positively charged. Moreover, it does not absorb ultraviolet light, as does protoporphyrin, its precursor. Thus, where these molecules behave similarly, as in the activation of Factor XIIdependent pathways, it seems likely that the effect is due to the negative charge of the peripheral carboxyl groups and not to an interaction involving the iron atom. In fact, one might expect hematin to quench a reaction due to absorbed ultraviolet light. This is suggested by the finding that hematin fails to activate complement after prolonged irradiation, while the other porphyrins studied have graded responses.

The observation that hematin can activate Factor XIIdependent pathways may in part explain the coagulopathy associated with administration of hematin to patients with inducible porphyria. Protoporphyrin and hematin may potentiate the autoactivation of Factor XII by virtue of their overall negative charge. However, it has recently been reported that hematin can, in the presence of oxygen and 2-mercaptoethanol, mediate oxidative degradation of certain proteins, which suggests that other mechanisms of activation may exist (29).

The activation by protoporphyrin of both the contact system of human plasma and, on exposure to long wave ultraviolet light, the classical pathway of the complement system, may either initiate or contribute to the severity of the skin injury observed in patients with protoporphyria and/or porphyria cutanea tarda. In this connection, it has been observed that the extravascular compartment of the skin of guinea pigs contains significant quantities of Factor XII that is functionally and immunochemically indistinguishable from plasma Factor XII (30). Since the proportion of the various heme precursors in these diseases varies, further investigation into the mechanisms whereby porphyrins activate the contact and complement systems and cause tissue injury, may shed light on the pathological changes observed in the various porphyrias.

\section{Acknowledgments}

The authors are indebted to Ms. Elba Perez for technical assistance, to Mrs. Barbara Whyte for help in manuscript preparation, and to Dr. Ralph Nachman for helpful suggestions.

This paper is supported in part by National Institutes of Health grants AM 30664 and AM 30203, and NHLBI grants 18828 and 23704-05.

\section{References}

1. Kappas, A., S. Sassa, and K. E. Anderson. 1983. In The Metabolic Basis of Inherited Disease. J. B. Stanbury, J. B. Wyngaarden, and D. S. Fredrickson, editors. McGraw-Hill Inc., New York. 13011384. 
2. Elder, H. H. 1982. Enzymatic defects in porphyria: an overview. Seminars Liver Dis. 2:87-99.

3. Gigli, I., A. A. Schothorst, N. A. Soter, and M. A. Pothak. 1980. Erythropoietic protoporphyria: photoactivation of the complement system. J. Clin. Invest. 66:517-522.

4. Lim, H. W., H. D. Perez, I. Goldstein, and I. Gigli. 1981 Complement-derived chemotactic activity is generated in human serum containing uroporphyrin after irradiation with $\mathbf{4 0 5}$ nanometers of light. J. Clin. Invest. 67:1072-1077.

5. Lim, H. W., H. D. Perez, M. Poh-Fitzpatrick, I. M. Goldstein, and I. Gigli. 1981. Generation of chemotactic activity in serum from patients with erythropoietic protoporphyria and porphyria cutanea tarda. N. Engl. J. Med. 304:212-216.

6. Lim, H. W., M. B. Poh-Fitzpatrick, and I. Gigli. 1984. Activation of the complement system in patients with porphyrias after irradiation in vivo. J. Clin. Invest. 74:1961-1965.

7. Lamon, J. M., B. C. Frykholm, R. A. Hess, and D. P. Tschudy. 1979. Hematin therapy for acute porphyria. Medicine. 58:252-269.

8. Morris, D. L., M. D. Dudley, and R. D. Pearson. 1981. Coagulopathy associated with hematin treatment for acute intermittent porphyria. Ann. Int. Med. 95:700-701.

9. Ellfolk, N., and G. Sievers. 1966. Thin layer chromatography of free porphyrins. J. Chromatogr. 25:373-379.

10. Collier, G. S., J. H. Pratt, C. R. DeWet, and C. F. Tshabalala. 1979. Studies on haemin in dimethyl sulphoxide/water mixtures. Biochem. J. 179:281-289.

11. Becker, C. G., and T. Dubin. 1977. Activation of factor XII by tobacco glycoprotein. J. Exp. Med. 146:457-467.

12. Ogston, D., N. B. Bennett, C. M. Ogston, and O. D. Ratnoff. 1971. The assay of a plasma component necessary for the generation of a plasminogen activator in the presence of Hageman factor. Br. J. Haematol. 20:209-216.

13. Silverberg, M., J. T. Dunn, L. Garen, and A. P. Kaplan. 1980. Autoactivation of human Hageman factor: demonstration using a synthetic substrate. J. Biol. Chem. 255:7281-7286.

14. Claeson, G., P. Friberger, M. Know, and E. Eriksson. 1978. Methods for determination of prekallikrein in plasma, glandular kallikrein and urokinase. Haemostasis. 7:76-78.

15. Hojima, Y., J. V. Pierce, and J. J. Pisano. 1980. Hageman factor inhibitor in corn seeds: purification and characterization. Thromb. Res. 20:149-162.
16. Bruning, J. L., and B. L. Kintz. 1977. In Computational Handbook of Statistics. Scott, Foresman, and Co., Glenview, IL. Second ed. 113-116.

17. Truedsson, L., A. G. Sjöholm, and A.-B. Laurell. 1981. Screening for deficiencies in the classical and alternative pathways of complement by hemolysis in gel. Acta Microbiol. Scand. 89:161-166.

18. Laurell, C.-B. 1965. Antigen-antibody crossed electrophoresis. Anal. Biochem. 10:358-361.

19. Duff, G. W., and E. Atkins. 1982. The inhibitory effect of polymyxin B on endotoxin-induced endogenous pyrogen production. J. Immunol. Methods. 52:333-340.

20. Griffin, J. H. 1979. Role of surface in surface dependent activation of Hageman factor (blood coagulation factor XII). Proc. Natl. Acad. Sci. USA. 75:1998-2002.

21. Miller, G., M. Silverberg, and A. P. Kaplan. 1980. Autoactivation of human Hageman factor. Biochem. Biophys. Res. Commun. 92:803810.

22. Silverberg, M., and A. P. Kaplan. 1982. Enzymatic activities of activated and zymogenic forms of human Hageman factor (factor XII). Blood. 60:64-70.

23. Tankersley, D. L., and J. Finlayson. 1984. Kinetics of activation and autoactivation of human factor XII. Biochem. J. 23:273-279.

24. Morrison, D. C., and C. G. Cochrane. 1974. Direct evidence for Hageman factor (factor XII) activation by bacterial lipopolysaccharides (endotoxins). J. Exp. Med. 140:797-811.

25. Green, D., N. Reynolds, J. Klein, H. Kohn, and C.-H. Ts'ao. 1983. The inactivation of hemostatic factors by hematin. J. Lab. Clin. Med. 102:361-369.

26. Lim, H. W., H. Novotny, and I. Gigli. 1983. Role of complement and polymorphonuclear cells in dimethyl chlortetracycline-induced phototoxicity in guinea pigs. J. Clin. Invest. 71:1326-1335.

27. Seery, V. L., W. T. Morgan, and U. Muller-Eberhard. 1975. Interaction of rabbit hemopexin with rose bengal and photooxidation of the rose bengal-hemopexin complex. J. Biol. Chem. 250:6439-6444.

28. Brown, S. B., M. Shillcock, and P. Jones. 1976. Equilibrium and kinetic studies of the aggregation of porphyrins in aqueous solution. Biochem. J. 153:279-285.

29. Aft, R. L., and G. C. Mueller. 1984. Hemin mediated oxidative degradation of proteins. J. Biol. Chem. 259:301-305.

30. Yamamoto, T., and C. G. Cochrane. 1982. A protease-like permeability factor in guinea pig skin. Immunologic identity with plasma Hageman factor. Am. J. Pathol. 107:127-134. 